\title{
Neonatal Graves' Disease with Maternal Hypothyroidism
}

\author{
Gangaram Akangire, MD ${ }^{1,2}$ Alain Cuna, MD ${ }^{1,2}$ Charisse Lachica, MD ${ }^{1,2}$ Ryan Fischer, MD ${ }^{1,2}$ \\ Sripriya Raman, MD $^{1,2}$ Venkatesh Sampath, MBBS ${ }^{1,2}$
}

\author{
${ }^{1}$ Department of Pediatrics, Children's Mercy Kansas City, \\ Kansas City, Missouri \\ 2 Department of Pediatrics, University of Missouri-Kansas City School \\ of Medicine, Kansas City, Missouri
}

Am J Perinatol Rep 2017;7:e181-e184.

\begin{abstract}
Address for correspondence Gangaram Akangire, MD, MS, FAAP, Section of Neonatology, Children's Mercy Kansas City, 2401 Gillham Road, Kansas City, MO 64108 (e-mail: gakangire@cmh.edu).
\end{abstract}

\begin{abstract}
Keywords

- cholestasis

- failure to thrive

- hyperthyroidism

- Graves' disease

Neonatal Graves' disease presenting as conjugated hyperbilirubinemia is a diagnostic challenge because the differential includes a gamut of liver and systemic diseases. We present a unique case of neonatal Graves' disease in a premature infant with conjugated hyperbilirubinemia born to a mother with hypothyroidism during pregnancy and remote history of Graves' disease. Infant was treated with a combination of methimazole, propranolol, and potassium iodide for 4 weeks. Thyroid function improved after 8 weeks of treatment with full recovery of thyroid function, disappearance of thyroid-stimulating antibodies, and resolution of failure to thrive and conjugated hyperbilirubinemia. This case provides several clinical vignettes as it is a rare, severe, presentation of an uncommon neonatal disease, signs, symptoms, and clinical history presented a diagnostic challenge for neonatologists and endocrinologists, normal newborn screen was misleading, and yet timely treatment led to a full recovery.
\end{abstract}

Neonatal Graves' disease develops in only 1.5 to $2.5 \%$ of infants born to mothers with Graves' disease. ${ }^{1}$ Well-recognized signs and symptoms include goiter causing tracheal compression, hyperthermia, tachycardia, irritability, heart failure, hydrops, hypertension, diarrhea, and craniosynostosis. ${ }^{2,3}$ Since the individual symptoms and signs can be nonspecific, several etiologies including viral disease, sepsis, and metabolic and genetic disease should be considered. Gaining an accurate maternal history and high index of suspicion combined with obtaining critical laboratories are key to making the diagnosis. Accurate and timely diagnosis of Graves' disease is important to prevent significant neonatal morbidity and mortality, which can be present in up to $20 \%$ of cases. ${ }^{4,5}$ Further, recent reports suggest that delayed diagnosis and treatment can have adverse impact on neurocognitive outcomes. ${ }^{6,7}$ In this report, we discuss the importance of considering neonatal Graves' disease in the differential diagnosis for unexplained failure to thrive and neonatal cholestasis.

received

June 20, 2017

accepted after revision

July 30, 2017

\section{Case}

A 33-week, 1.54-kg (appropriate for gestational age, 11\%) male infant was born via vaginal delivery to a 38 -year-old G8P4Ab4L4 mother at a community hospital due to preterm labor. The pregnancy was complicated by maternal hypothyroidism diagnosed during the first trimester and treated with levothyroxine. Other prenatal laboratory tests were unremarkable. The mother received one dose of betamethasone. Rupture of membranes occurred 27 hours prior to delivery. Apgar scores were 8 and 9 at 1 and 5 minutes after birth, respectively. The infant was stable in room air and transferred to closest regional level III neonatal intensive care unit for further management.

On day of life (DOL) 3, the infant developed a purpuric "blueberry muffin" rash, prompting a work-up for cytomegalovirus, herpes simplex virus, and enterovirus infection that eventually was negative. The infant was also noted to have baseline tachycardia with heart rate (HR) ranging from 200 to

Copyright $\odot 2017$ by Thieme Medical Publishers, Inc., 333 Seventh Avenue, New York, NY 10001, USA. Tel: +1(212) 584-4662.
License terms

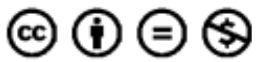

DOI https://doi.org/ 10.1055/s-0037-1606365. ISSN 2157-6998. 


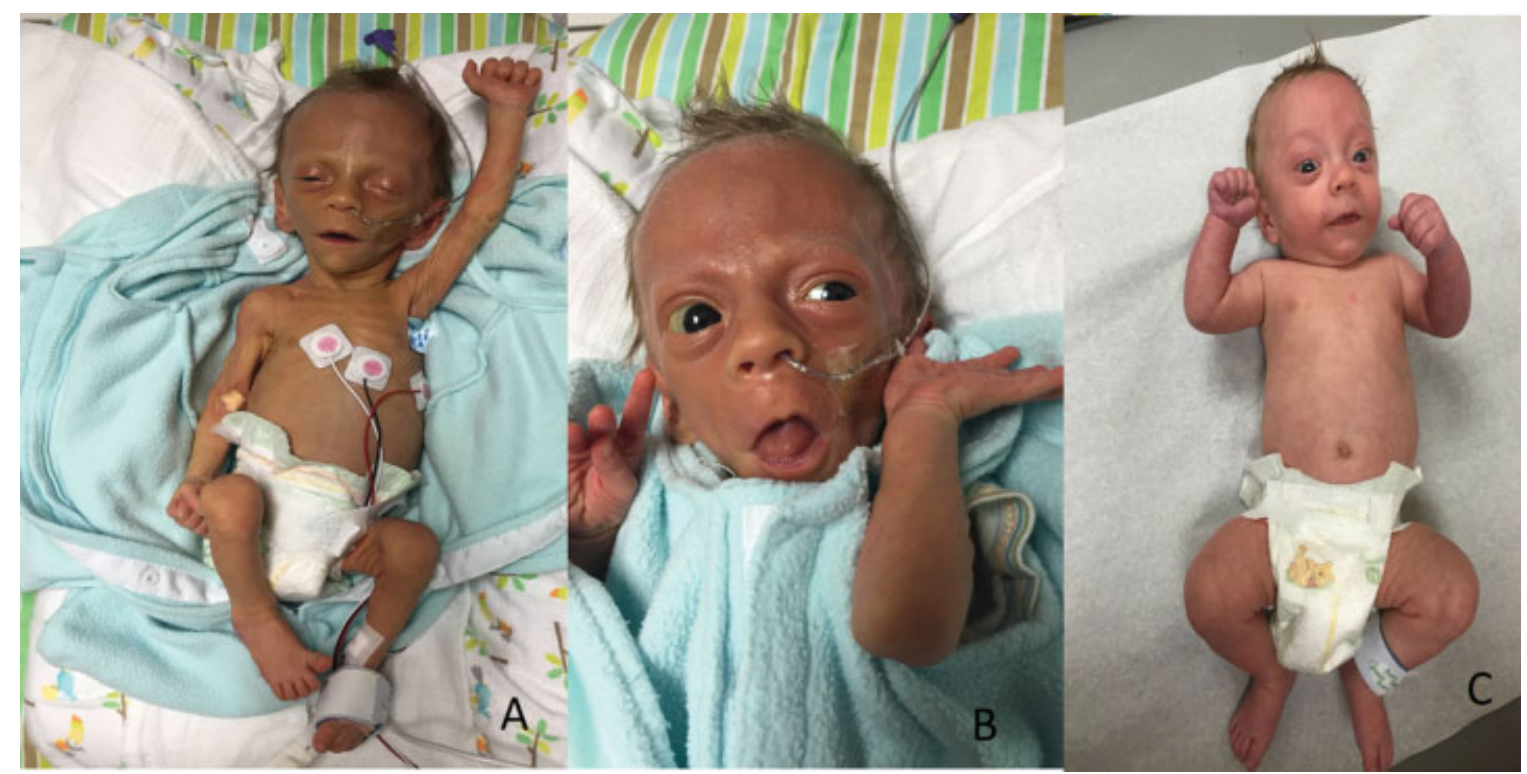

Fig. 1 (A) Severely emaciated infant with jaundice at 3 weeks of age despite full-volume high calorie enteral feeds. (B) Infant noted to have mild proptosis. (C) Infant after 2 months of diagnosis and treatment initiation.

230 beats/min, but an electrocardiogram showed normal sinus rhythm and an echocardiogram was within normal limits. On DOL 5, the infant appeared jaundiced on exam. Initial work-up showed direct bilirubin was elevated to $13.4 \mathrm{mg} / \mathrm{dL}$. Aspartate aminotransferase and alanine transaminase were also elevated, but abdominal and liver ultrasounds were normal. Infant was started on combination of parental and enteral nutrition. Enteral feeds were gradually advanced to full volume feeds, but despite adequate intake of $26 \mathrm{Kcal} / \mathrm{oz}$ formula at 160 $\mathrm{mL} / \mathrm{kg} / \mathrm{d}$, the infant exhibited poor weight gain. Direct bilirubin also remained elevated despite the infant being on full feeds. On DOL 20, the infant was transferred to the neonatal intensive care unit at our tertiary care center for further evaluation and management.

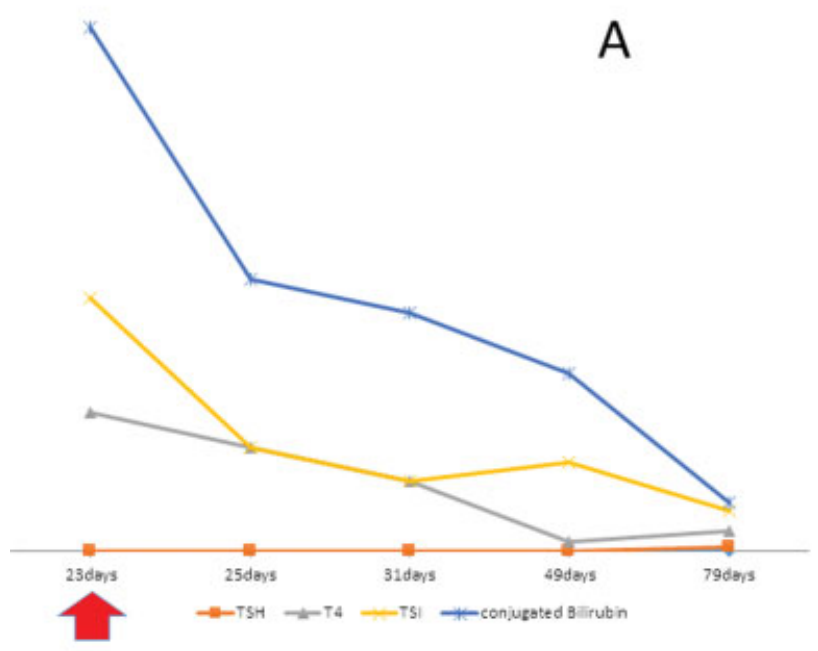

On admission physical exam, the infant was significantly emaciated (weight $<3 \%$ and Z-score of -2.5 on the Fenton chart) with mild hepatomegaly, jaundice, and intermittent tachycardia (HR of $200-230$ beats/min), and mild proptosis (-Fig. 1A, B). The infant also had a history of pale stools. His pulses were normal, and he had a quiet precordium. Multiple laboratories to evaluate cholestasis were obtained, including thyroid function tests ( - Fig. 2A). On DOL 21, thyroid function tests were abnormal, with markedly elevated free $\mathrm{T} 4$ (fT4) of $>7 \mathrm{ng} / \mathrm{dL}(0.8-1.9)$ and appropriately suppressed thyroid-stimulating hormone (TSH) of $<0.02 \mathrm{mcIU} / \mathrm{mL}$ (reference range: 0.35-7.6), suggesting thyrotoxicosis.

A detailed history from the mother revealed that she had Graves' disease 9 years ago, which resolved with radioactive

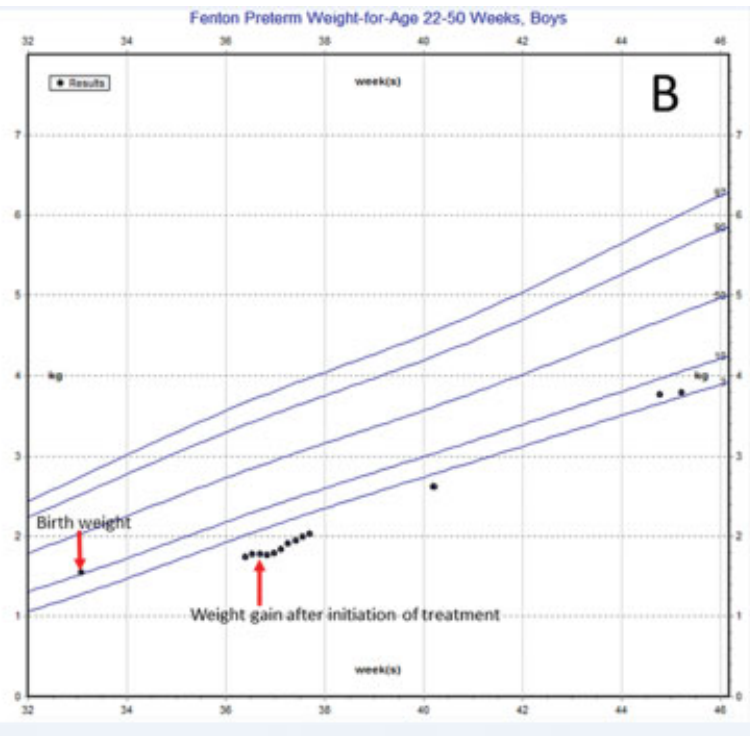

Fig. 2 (A) Gradual improvement of thyroid function tests over a 2-month period. Note the decline in TSI, conjugated bilirubin, and T4 levels. Note the return of innate thyroid function with slight increase in T4 and TSH after 49 days of life and stopping treatment. The red arrow indicates day of treatment initiation. (B) Gradual weight gain after treatment. TSH, thyroid-stimulating hormone; TSI, thyroid-stimulating immunoglobulins. 
ablation. The mother was lost to follow-up and did not see a doctor until this pregnancy, where she was found to have hypothyroidism and was started on levothyroxine. The medical record contained no mention of her previous history of Graves' disease, and thyroid-stimulating immunoglobulins (TSI) were not measured during pregnancy. Thyroid-stimulating immunoglobulins, which should be essentially undetectable in a normal newborn, were markedly elevated at 5.7 units/L in this infant, confirming the diagnosis of neonatal Graves' disease (-Fig. 2A). The infant was treated with one drop of potassium iodide daily for 5 days, and oral methimazole $1 \mathrm{mg} / \mathrm{kg} / \mathrm{d}$ and propranolol $2 \mathrm{mg} / \mathrm{kg} / \mathrm{d}$ for 4 weeks. He improved dramatically with treatment, with direct bilirubin down to $4.5 \mathrm{mg} / \mathrm{dL}$ after only 10 days of treatment. He was subsequently discharged home and had complete resolution of clinical signs and symptoms over 6 weeks with outpatient therapy and appropriate weight gain (-Figs. 1C and 2B).

\section{Discussion}

In this report, we describe an unusual case of neonatal Graves' disease presenting as neonatal cholestasis and failure to thrive in the setting of maternal hypothyroidism. The diagnosis of hypothyroidism in the mother obscured the clinical picture and made the diagnosis of neonatal Graves' disease more challenging. The remote maternal history of Graves' disease and radioactive ablation, which would have been helpful in raising the suspicion for neonatal Graves' disease, was also not readily evident and was probably unknown at the outlying hospital. Our case highlights the importance of obtaining an accurate maternal history, which should be reviewed and revisited with each newborn admission and transfer. Other disease processes beyond the hepatobiliary system should also be considered in the differential diagnosis of neonatal cholestasis, including metabolic, infectious, and endocrine processes.

We identified only four reports in the literature describing the relationship between maternal Graves' disease and neonatal cholestasis. ${ }^{8-11}$ Beroukhim et al, Varier et al, Khadora and Al Dubayee, and Loomba-Albrecht et al reported four term infants who presented with neonatal thyrotoxicosis and cholestasis in the setting of known maternal Graves' disease and hyperthyroidism. ${ }^{8-11}$ Unlike these previous reports, in our case, the infant was preterm and the mother was treated for hypothyroidism during pregnancy. We speculate that the clinical picture of neonatal hyperthyroidism in the setting of maternal hypothyroidism was due to high maternal TSH receptor antibodies (TRAb) or TSI levels that were sufficient to cause a hyperthyroid state in the fetus and neonate, but not high enough to activate the maternal thyroid tissue. It is also possible that although TRAb levels were high in the mother, there was insufficient thyroid tissue to respond and secrete T3 and $\mathrm{T} 4$ due to history of radioactive ablation.

It is well known that hypothyroidism can lead to unconjugated hyperbilirubinemia in a newborn, but conjugated hyperbilirubinemia is very uncommon with thyroid diseases. ${ }^{9}$ Loomba-Albrecht et al reported cholestasis as a presenting sign of neonatal Graves' disease, but the mother was treated with methimazole during pregnancy. ${ }^{9}$ Methimazole is also known to cause liver failure leading to cholestasis, which makes it unclear in this case report whether hyperthyroidism or methimazole led to cholestasis in this infant. ${ }^{9}$ Khadora and Al Dubayee describe that ammonia levels can also be elevated along with cholestasis in neonatal Graves' disease. ${ }^{10}$ Varier et al have described neonatal Graves' disease with cholestasis in a prenatally undiagnosed and untreated mother with Graves' disease. ${ }^{11}$ Postulated mechanisms for cholestasis in neonatal Graves' disease described in the literature include potential for thyroxine-driven free radical generation or fatty deposition leading to cholestasis, as well as congestive heart failure leading to cholestasis. ${ }^{12,13}$

The infant in our case had subtle signs of hyperthyroidism after birth as evidenced by intermittent tachycardia as high as 230 beats/min and poor weight gain despite feeding high calorie Eq. (26 Kcal/oz at $\sim 200 \mathrm{~mL} / \mathrm{kg} / \mathrm{d}$ ). However, the diagnosis was made difficult because neonatal hyperthyroidism presents with several nonspecific symptoms that are also common in premature infants, such as feeding difficulties, diarrhea, irritability, poor weight gain, hyperthermia, tachycardia, and tachypnea. ${ }^{1}$ Our infant also presented with cholestasis with conjugated bilirubin as high as $13.4 \mathrm{mg} / \mathrm{dL}$, which directed the initial work-up into possible infectious and metabolic hepatic diseases, as cholestasis is a very rare presentation of neonatal Graves' disease. ${ }^{13}$ The diagnosis of hypothyroidism in the mother and an unknown remote history of maternal Graves' disease also contributed to a challenging clinical picture. A normal newborn screen, which is based on high TSH levels, can detect only hypothyroidism and not hyperthyroidism.

If maternal history of Graves' disease is known, maternal TRAb levels should be measured during pregnancy. Maternal TRAb levels $>4$-fold of the reference range have a high positive predictive value of $40 \%$ to detect developing neonatal hyperthyroidism, and levels $<4$-fold of reference range have a negative predictive value of $100 \%{ }^{1}$ It has also been reported that maternal TRAb levels can be positive during pregnancy even with history of radioactive ablation of thyroid gland. ${ }^{12}$ Neonatal TRAb levels should be checked on DOL 1 followed by fT4 and TSH on DOL 3. ${ }^{1}$ If the infant is symptomatic and has abnormal laboratory values confirming hyperthyroidism, methimazole and propranolol should be initiated. ${ }^{1}$ In infants with hemodynamic instability, potassium iodide should be considered for 3 to 5 days until methimazole levels in the blood are therapeutic. $^{1}$ The infant in our case was treated for 4 weeks, but in most cases, treatment is required for 1 to 2 months. ${ }^{1}$ Neonatal Graves usually resolves in 6 months but is highly dependent on initial TRAb levels and can take up to 12 months to resolve in rare cases. ${ }^{1}$

\section{Conclusion}

In summary, we describe an unusual case of neonatal Graves' disease presenting with failure to thrive and cholestasis in the setting of resolved maternal Graves' disease due to radioablation therapy resulting in hypothyroidism. Maternal 
euthyroid status did not prevent transplacental transfer of TRAb, which subsequently led to fetal and neonatal hyperthyroidism. A newborn screen cannot be relied upon for detecting neonatal Graves' disease because it is set to identify the more common condition of primary hypothyroidism where neonates have elevated TSH levels. A meticulous maternal history, high index of suspicion, and appropriate testing for thyroid function and TRAb or TSI in the mother during the third trimester and in the infant after birth are essential to make a prompt diagnosis. Appropriate treatment results in resolution of symptoms and is likely to decrease the number of invasive tests and optimize longterm outcome.

\section{Conflict of Interest}

None.

\section{Acknowledgements}

The authors thank medical writing center at the Children's Mercy for their support in reviewing the article.

\section{References}

1 van der Kaay DC, Wasserman JD, Palmert MR. Management of neonates born to mothers with Graves' disease. Pediatrics 2016; 137(04):e20151878

2 Zimmerman D. Fetal and neonatal hyperthyroidism. Thyroid 1999;9(07):727-733

3 Polak M, Van Vliet G. Therapeutic approach of fetal thyroid disorders. Horm Res Paediatr 2010;74(01):1-5
4 Mitsuda N, Tamaki H, Amino N, Hosono T, Miyai K, Tanizawa O. Risk factors for developmental disorders in infants born to women with Graves disease. Obstet Gynecol 1992;80(3 Pt 1): 359-364

5 Peleg D, Cada S, Peleg A, Ben-Ami M. The relationship between maternal serum thyroid-stimulating immunoglobulin and fetal and neonatal thyrotoxicosis. Obstet Gynecol 2002;99(06):1040-1043

6 Smit BJ, Kok JH, Vulsma T, Briët JM, Boer K, Wiersinga WM. Neurologic development of the newborn and young child in relation to maternal thyroid function. Acta Paediatr 2000; 89(03):291-295

7 Messer PM, Hauffa BP, Olbricht T, Benker G, Kotulla P, Reinwein D. Antithyroid drug treatment of Graves' disease in pregnancy: longterm effects on somatic growth, intellectual development and thyroid function of the offspring. Acta Endocrinol (Copenh) 1990; 123(03):311-316

8 Beroukhim RS, Moon TD, Felner EI. Neonatal thyrotoxicosis and conjugated hyperbilirubinemia. J Matern Fetal Neonatal Med 2003;13(06):426-428

9 Loomba-Albrecht LA, Bremer AA, Wong A, Philipps AF. Neonatal cholestasis caused by hyperthyroidism. J Pediatr Gastroenterol Nutr 2012;54(03):433-434

10 Khadora MM, Al Dubayee M. Neonatal Graves' disease with unusual metabolic association from presentation to resolution. BMJ Case Rep 2014;2014:1-5

11 Varier RU, Jensen MK, Adams CJ, Book LS. Neonatal cholestasis caused by undiagnosed maternal Graves' disease. ACG Case Rep J 2014;2(01):58-60

12 Papendieck P, Chiesa A, Prieto L, Gruñeiro-Papendieck L. Thyroid disorders of neonates born to mothers with Graves' disease. J Pediatr Endocrinol Metab 2009;22(06):547-553

13 Dryden C, Simpson JH, Hunter LE, Jackson L. An unusual cause of neonatal coagulopathy and liver disease. J Perinatol 2007;27(05): 320-322 